\title{
Nucleon and $\Delta$ isobar in a strong magnetic field
}

\author{
Ulugbek Yakhshiev, ${ }^{1, *}$ Hyun-Chul Kim, ${ }^{1,2,3, \dagger}$ and Makoto Oka ${ }^{2, \dagger}$ \\ ${ }^{1}$ Department of Physics, Inha University, Incheon 22212, Republic of Korea \\ ${ }^{2}$ Advanced Science Research Center, Japan Atomic Energy Agency, \\ Shirakata, Tokai, Ibaraki 319-1195, Japan \\ ${ }^{3}$ School of Physics, Korea Institute for Advanced Study (KIAS), Seoul 02455, Republic of Korea
}

(Received 3 February 2019; published 28 March 2019)

\begin{abstract}
We investigate the static properties of the nucleon in the presence of strong magnetic fields and discuss the consequent changes of the nucleon structure, based on the Skyrme model. The results show that at large values of the magnetic field $\left(\sim 10^{17}\right.$ to $\left.10^{18} \mathrm{G}\right)$, which is supposed to appear in heavy-ion collision experiments at RHIC energies, the soliton starts to deviate from the spherically symmetric form and its size starts to change. At extremely large values of the magnetic field $\left(\sim 10^{19} \mathrm{G}\right)$, which may be found at the LHC experiments, the soliton becomes more compact than in free space. The results also show that in the presence of the external magnetic field, the mass of the nucleon tends to increase in general and the mass degeneracy of the $\Delta$ isobars from isospin symmetry will be lifted. We also discuss the changes in the mass difference between the $\Delta$ and the nucleon, $\Delta m_{\Delta \mathrm{N}}$, due to the influence of the external magnetic field. We find that $\Delta m_{\Delta \mathrm{N}}$ increases as the strength of the magnetic field grows.
\end{abstract}

DOI: 10.1103/PhysRevD.99.054027

\section{INTRODUCTION}

Understanding how hadrons are modified in the presence of various external fields is an important topic in contemporary physics of hadrons. In particular, it is of great interest to investigate how the nucleon undergoes change in a strong magnetic field, since it provides certain information on both compact astrophysical objects and ultrarelativistic heavy-ion collision (URHIC), which unveils the nature of matter in the early Universe. A very strong magnetic field may exist in a magnetar in which the magnetic field reaches an order of $B_{M} \sim\left(10^{11}-10^{15}\right) \mathrm{G}$ $[1,2] .{ }^{1}$ Even stronger magnetic fields $\left(\sim 10^{16}\right.$ to $\left.10^{17} \mathrm{G}\right)$ may be found in the cosmological $\gamma$-ray bursts [3-5]. However, one can create even much stronger magnetic fields in the course of relativistic heavy-ion collisions [6]. At the Relativistic Heavy-Ion Collider (RHIC), the magnetic field could reach $B_{M} \sim 3 \times 10^{18} \mathrm{G}$ and it may even rise to $B_{M} \sim 10^{19} \mathrm{G}$ at the Large Hadron Collider

*yakhshiev@inha.ac.kr

hchkim@inha.ac.kr

\#oka@post.j-parc.jp

${ }^{1}$ Here $B_{M}$ denotes the strength of a magnetic field. Note that we adopt the gauss $(\mathrm{G})$ as the unit of the strength of the magnetic field. $1 \mathrm{G}$ corresponds to $2 \times 10^{-20} \mathrm{GeV}^{2}$.

Published by the American Physical Society under the terms of the Creative Commons Attribution 4.0 International license. Further distribution of this work must maintain attribution to the author(s) and the published article's title, journal citation, and DOI. Funded by SCOAP.
(LHC) [7-15]. Although such an extremely strong magnetic field exists only during a very short period of time, it may bring about the distortion of hadrons and may change their properties greatly.

There has been already a great deal of theoretical works on modifications of hadrons under the influence of strong magnetic fields [16-31]. However, while they mainly concentrate on the modification of light and heavy meson properties in the presence of the strong magnetic fields, there are only few works on the changes of properties of the nucleon [31,32]. Since Refs. [31,32] aim at describing the neutron stars, they focus only on the modification of the neutron in the strong magnetic fields. In the present work, we will investigate the modifications of the nucleon and $\Delta$ isobar properties in the presence of the strong magnetic fields within the framework of a chiral soliton approach.

The approach provides a simple but effective way of describing the structure of the nucleon. The main idea arises from the seminal papers by Witten [33-35]. In the limit of $N_{c} \rightarrow \infty$ ( $N_{c}$ as the number of colors), the mass of the nucleon is proportional to $N_{c}$ whereas its width is of order $\mathcal{O}(1)$, which indicates that the meson fluctuations can be neglected. In this picture, a baryon arises as a topological chiral soliton that is called skyrmion [36,37]. The nucleon as a chiral soliton is naturally an extended object, so that one can examine how the nucleon undergoes changes when a very strong magnetic field is exerted on it. A theoretical method has been developed over years, the environment surrounding the nucleon being treated collectively. It has been successfully applied to the description of the nucleon 
in nuclear medium [38-43], the nucleon in finite nuclei [44], the properties of nuclear matter [45] and even to the explanation of properties of atomic nuclei $[46,47]$. The similar theoretical tool can be utilized for describing the nucleon in the strong magnetic field.

From a technical point of view, the nucleon in an external magnetic field is very similar to the situation when a skyrmion is embedded into an isospin asymmetric nuclear environment [45]. In general, one may expect that the magnetic field will change the nucleon properties less than the effects of isospin symmetry breaking. However, when it comes to the very strong magnetic fields that reach the level of URHICs at the LHC, the effects from the magnetic fields may become sizable. In this case, they may also play a crucial role in describing the evolution of the universe at an early stage $[48,49]$. Moreover, such strong magnetic fields will reveal certain novel features relevant to the structure of the nucleon.

Depending on a specific configuration of the external magnetic field, one may further expect possible nonspherical deformations of the skyrmion in isospin and ordinary spaces $[44,46,47]$ from the spherically symmetric hedgehog form corresponding to the skyrmion in free space $[37,50]$. In this sense, the situation becomes even more interesting if the nucleon properties are studied in the presence of external isospin asymmetric nuclear environment that actually creates the strong magnetic field, that is, if the nucleon is located inside compact stellar objects in the presence of strong magnetic fields. The corresponding investigation can naturally be performed by generalizing the approach developed in Refs. [44-47] in the presence of an additional external magnetic field. However, we will concentrate only on the external magnetic field for simplicity and leave more general and complex studies as future works.

In the present work, we consider the homogeneous magnetic field oriented along the axis of quantization. This choice allows us to consider axially symmetric solutions of the classical equation of motion for the soliton instead of a complicated situation where the soliton has totally an asymmetric form. Then we can employ the technique developed already for asymmetric nuclear environment $[44,46,47]$. Nevertheless, it is necessary to note that in the present work there will be some differences at the Lagrangian level due to the nature of the external magnetic field influencing the properties of the nucleon under consideration. In Refs. $[44,46,47]$ the effect of environment on the skyrmion properties was introduced by means of the density functions, based on phenomenological information taken from mesonic atoms at low densities. Further modifications were achieved by introducing another density functions into the Lagrangian and relating them to the properties of nuclear matter near the saturation point $\rho_{0} \approx 0.16 \mathrm{fm}^{-3}$ [45]. In the present work, the external magnetic field will be introduced by taking into account the U(1) gauge field into the original effective chiral Lagrangian [51].

The present paper is organized as follows: In the next Sec. II, we briefly discuss the Lagrangian of the model and the axially symmetric ansatz for the solutions of field equations. In Sec. III, we explain the variational method for the problem and discuss the parametrizations of profile functions. We also discuss the minimization process and present the classical results. Then we discuss how the baryon charge distribution is changed to a spheroidal form under the influence of the magnetic field. In Sec. IV, we show how to quantize the spheroidal skyrmion and discuss the changes of the nucleon properties in the magnetic field. In the last Sec. V we summarize the present results, draw conclusions, and give future outlook. The explicit expressions of the mass functional and the moments of inertia of the spheroidal skyrmion can be found in the Appendix.

\section{LAGRANGIAN AND ANSATZ}

We start with the effective chiral Lagrangian, incorporating explicit chiral symmetry breaking [50]

$$
\begin{aligned}
\mathcal{L}= & -\frac{F_{\pi}^{2}}{16} \operatorname{Tr} L_{\mu} L^{\mu}+\frac{1}{32 e^{2}} \operatorname{Tr}\left[L_{\mu}, L^{\nu}\right]^{2} \\
& +\frac{F_{\pi}^{2} m_{\pi}^{2}}{16} \operatorname{Tr}\left[U+U^{\dagger}-2\right],
\end{aligned}
$$

where the first term is called the Weinberg term and the second one was originally introduced by Skyrme [36], which is also known as the Gasser-Leutwyler term in the large $N_{c}$. The chiral current $L_{\mu}$ is defined as $L_{\mu}=U^{+} \partial_{\mu} U$, where the $\mathrm{SU}(2)$ unitary matrix $U=\exp \left\{2 i \tau_{a} \pi_{a} / F_{\pi}\right\}$ is expressed in terms of the Cartesian isospin-components of the pion field $\pi_{a}(a=1,2,3) \cdot \tau^{a}$ stand for the Pauli matrices in isospin space. There are three input parameters, i.e., the pion decay constant $F_{\pi}=108.783 \mathrm{MeV}$, the Skyrme parameter $e=4.854$, and the pion mass $m_{\pi}=134.977 \mathrm{MeV}$, which are chosen in such a way that the model properly reproduces the experimental data on the masses of the proton and neutron with breakdown of isospin symmetry taken into account (for the details, see Refs. [46,47]).

In order to consider the effects of the external magnetic field we introduce the U(1) gauge field into the Lagrangian of Eq. (1). So, the ordinary derivative is replaced by the covariant one given in the form of

$$
D_{\mu} U=\partial_{\mu} U+i q_{e} A_{\mu}[Q, U]
$$

where $q_{e}$ denotes the electric charge and $A_{\mu}$ stands for the electromagnetic four-vector potential (e.g., see Ref. [52]). Here the charge operator in the $\mathrm{SU}(2)$ framework is defined as 


$$
Q=\frac{1}{6} \mathbb{I}+\frac{1}{2} \tau_{3}
$$

As mentioned above, we introduce the homogeneous magnetic field along the quantization axis or the $z$ direction $\boldsymbol{B}_{M}=\left(0,0, B_{M}\right)$, so we fix correspondingly the gauge of $A^{\mu}$ as follows

$$
A^{\mu}=\left(0,-\frac{1}{2} y B_{M}, \frac{1}{2} x B_{M}, 0\right)
$$

When the magnetic field is absent, the hedgehog ansatz is imposed to be a spherically symmetric hedgehog form $U=\exp \{i \boldsymbol{i} \cdot \boldsymbol{n} P(r)\}$, where the unit vector in isospin space is chosen as a normal vector $\boldsymbol{n}$ in ordinary three dimensional space. However, the ansatz for the skyrmion in the presence of the magnetic field may be deformed in the isospin and ordinary spaces deviating from the original spherical form in the absence of external fields. The most general form of the ansatz, which takes into account all possible deformations, can be represented as

$$
U(\boldsymbol{r})=\exp \{i \boldsymbol{\tau} \cdot \boldsymbol{N}(\boldsymbol{r}) P(\boldsymbol{r})\}
$$

where the normal vector in isospin space is expressed as

$$
\boldsymbol{N}=\left(\begin{array}{c}
\sin \Theta(r, \theta, \varphi) \cos \Phi(r, \theta, \varphi) \\
\sin \Theta(r, \theta, \varphi) \sin \Phi(r, \theta, \varphi) \\
\cos \Theta(r, \theta, \varphi)
\end{array}\right)
$$

in terms of two profile functions, $\Theta(r, \theta, \varphi)$ and $\Phi(r, \theta, \varphi)$. These two profile functions and $P(r, \theta, \varphi)$ describing the spatial extension of the pion fields will depend on all three (radial, polar and azimuthal) variables. ${ }^{2}$ Since we choose the magnetic field along the $z$ direction, we have an axial symmetry, so the profile functions $P$ and $\Theta$ become independent of the azimuthal angle $\varphi$, and the third profile function $\Phi$ can be selected as $\varphi$. Thus, one has the following axially symmetric ansatz

$$
P=P(r, \theta), \quad \Theta=\Theta(r, \theta), \quad \Phi=\varphi
$$

which will be used in the present work.

\section{CLASSICAL SOLITON MASS AND PARAMETRIZATIONS OF PROFILE FUNCTIONS}

Using the configuration given in Eqs. (5)-(7), one can find the mass of the static soliton $M$ in the presence of the static magnetic field $\boldsymbol{B}_{M}$ along the $z$ direction. The mass functional $M[P, \Theta]$ is explicitly written by Eq. (A2) in the Appendix. The field equations of the soliton can be derived

\footnotetext{
${ }^{2}$ In the present work we perform all calculations in the spherical coordinate system.
}

by variation of $M$ with respect to $P$ and $\Theta$. Since their expressions are rather lengthy and will not be used here, we will not present them in this work. In fact, they are coupled second-order partial differential equations of the following type $^{3}$

$$
\begin{gathered}
g\left(P_{r r}, P_{\theta \theta}, P_{r}, P_{\theta}, \Theta_{r}, \Theta_{\theta}, P, \Theta\right)=0, \\
h\left(\Theta_{r r}, \Theta_{\theta \theta}, \Theta_{r}, \Theta_{\theta}, P_{r}, P_{\theta}, \Theta, P\right)=0,
\end{gathered}
$$

and the boundary conditions are determined by the baryon number, i.e., $B=1$ in the present work. The baryon number of the axially deformed hedgehog configuration is given by the following expression

$$
B=-\frac{1}{\pi} \int_{0}^{\infty} d r \int_{0}^{\pi} d \theta\left(P_{r} \Theta_{\theta}-P_{\theta} \Theta_{r}\right) \sin ^{2} P \sin \Theta .
$$

Since we will use the variational method developed in Ref. [46], we will not write the explicit expression of the solitonic field equations, as mentioned previously. This will simplify all unnecessary technical complexities.

However, in order to clarify the form of trial functions to be used for a minimization process, let us for the moment ignore the nonspherical deformation effects and assume that the soliton has a spherical form even if it is affected by the magnetic field. Then the equation of motion becomes an ordinary but nonlinear differential equation. For our purpose, we will rather concentrate on its linear approximation $(r \rightarrow \infty)$ that yields the following form

$$
\begin{aligned}
& P^{\prime \prime}(r)+\frac{2}{r} P^{\prime}(r)-\frac{2}{r^{2}} P(r) \\
& \quad-\left(m_{\pi}^{2}+\frac{2 q_{e} B_{M}}{3}\right) P(r)-\frac{2\left(q_{e} B_{M} r\right)^{2}}{15} P(r)=0 .
\end{aligned}
$$

Note that the last two terms contribute differently, depending on whether the magnetic field is strong or weak. They will bring about interesting consequences and will play a key role in understanding the present results later.

In general, Eq. (9) has a Gaussian form of the solution

$$
\begin{aligned}
P(r) \sim & \frac{1}{2^{1 / 4} r^{2}} \exp \left\{-\frac{q_{e} B_{M} r^{2}}{\sqrt{30}}\right\} \\
& \times U\left(\frac{-3+\sqrt{30}}{12}+\frac{\sqrt{30} m_{\pi}^{2}}{8 q_{e} B_{M}},-\frac{1}{2} ; \sqrt{\frac{2}{15}} q_{e} B_{M} r^{2}\right),
\end{aligned}
$$

where $U(a, b ; c)$ is the confluent hypergeometric function of the second type. However, if $m_{\pi}^{2} \gg q_{e} B_{M}$, then one can ignore the quadratic term in $B_{M}$ of Eq. (9), keeping in mind that the soliton is localized at the finite region, i.e., even if $r$

\footnotetext{
${ }^{3}$ For the definitions of $F_{r}, \Theta_{\theta}$ etc., see the Appendix.
} 
is large, the last term in Eq. (9) is not important due to the localization of solution. Then the corresponding solution takes the Yukawa-type form

$$
P(r) \sim \frac{1+A r}{r^{2}} e^{-A r}, \quad A=\left(m_{\pi}^{2}+\frac{2}{3} q_{e} B_{M}\right)^{1 / 2} .
$$

We will return to the consequences arising from these two different behaviors of the solutions, when we discuss the results. Having analyzed the characteristics of the solutions at this stage, we are able to choose the most appropriate forms of the trial profile functions $P$ and $\Theta$.

As a result, we can apply the following approximations for the spheroidal solutions

$$
\begin{aligned}
P(r, \theta)= & 2 \arctan \left\{\frac{r_{0}^{2}}{r^{2}}(1+A r)[1+u(\theta)]\right\} \\
& \left.\times \exp \left\{-\beta_{0} A r-\beta_{1} q_{e} B_{M} r^{2}\right)\right\}, \\
\Theta(r, \theta)= & \theta+\zeta(r, \theta),
\end{aligned}
$$

where $r_{0}, \beta_{0}$ and $\beta_{1}$ are variational parameters. ${ }^{4}$ The functions $u$ and $\zeta$ satisfy the inequalities $|u|<1$ and $|\zeta|<1$ in the regions $r \in[0, \infty)$ and $\theta \in[0, \pi]$. Thus, the trial function in Eq. (12) correctly reproduces the asymptotic forms of the solutions for both the weak and strong magnetic fields, and provides the smooth transitions between these two different cases. Furthermore, following the ideas of Ref. [44], we use for the function $u$ the following parametrization

$$
u(\theta)=q_{e} B_{M} \sum_{n=1}^{\infty} \gamma_{n} \cos ^{n} \theta,
$$

where the set $\left\{\gamma_{n}\right\}$ consists of variational parameters in addition to those three mentioned previously. In the parametrization of Eq. (14), the cosine functions are chosen to maintain the periodicity in $\theta$. Similarly, $\zeta$ can be selected as

$$
\zeta(r, \theta)=q_{e} B_{M} r e^{-\delta_{0}^{2} r^{2}} \sum_{n=1}^{\infty} \delta_{n} \sin (2 n \theta),
$$

where the set $\left\{\delta_{n}\right\}$ contains the remaining part of all the variational parameters in the present work. The prefactor " $q_{e} B_{M}$ " in Eqs. (14) and (15) is introduced from the proper limiting consideration and will smooth the variational process.

Note that the arguments of the sine functions in Eq. (15) are picked out to be a multiple of $2 \theta$ in order to avoid singularities given in the form " $\sin \Theta / \sin \theta$," which can be found in the mass functional $M[P, \Theta]$. Furthermore, the $r$

\footnotetext{
${ }^{4}$ We note that the parametrization in Eqs. (12) and (13) are done in a most general form and indicates the different field regimes during our variational calculations in a natural way.
}

dependence of $\zeta$ is singled out such that the equalities $\Theta(0, \theta)=\theta$ and $\Theta(\infty, \theta)=\theta$ are reproduced correctly. The mass functional will be easily extremized in terms of the trial functions given in Eqs. (12)-(15), and $B=1$ condition will be naturally satisfied.

We want to mention that, in order to keep the minimization process with high accuracy, it is enough to consider only few terms in the trial functions (14) and (15). Furthermore, the current situation is in a more symmetric level than the case in which the nucleon is located in a finite nucleus at a given distance from its center. More specifically, when the nucleon is located inside the finite nucleus, the values of the profile functions $P$ and $\Theta$ with the polar angle given in $\theta \in[0, \pi / 2]$ are different from those with $\theta \in[\pi / 2, \pi]$. This is due to the fact that the external field, which is expressed by the density distribution function of the external system, depends on the radial distance from the center of the nucleus (see Ref. [46]). On the other hand, the present case is symmetric under the change of the polar angles from $\theta \in[0, \pi / 2]$ to $\theta \in[\pi / 2, \pi]$, because the external magnetic field is homogeneously exerted along the $z$ direction. Therefore, the symmetry in the polar angle brings about $\gamma_{2 n-1}=0(n=$ $1,2, \ldots)$ among $\left\{\gamma_{n}\right\}$ in Eq. (14).

We perform the variational calculation by minimizing the complete energy functional given in Eqs. (A2) and (A3), using the trial profile functions given in Eqs. (12) and (13). This approach is rather accurate, because both the solutions near the origin $(r \rightarrow 0)$ and asymptotic region $(r \rightarrow \infty)$ are properly given. The variational parameters introduced above connect smoothly the solution near the origin with the asymptotic one, which reproduces almost the exact solutions. For example, in the case of a free nucleon, we obtain almost the same results by either using the variational approach or directly solving the differential equations. Both the results differ within 1\% (e.g., see Table 1 of Ref. [46]). In the present work, the same level of high accuracy is achieved.

Figure 1 draws the results for the change of the classical soliton mass due to the external magnetic field, i.e., $M\left(B_{M}\right)-M(0)$, where $M\left(B_{M}\right)$ and $M(0)$ denote respectively the values of the mass obtained with and without the magnetic field exerted. The mass of the classical soliton remains constant till the strength of the magnetic field reaches around $10^{17} \mathrm{G}$. However, as the magnetic field gets stronger than $10^{17} \mathrm{G}$, the value of the soliton mass starts to increase slowly till $B_{M} \approx 10^{18} \mathrm{G}$. If one raises the magnitude of the magnetic field, then the soliton mass starts to rise rather rapidly. When the magnitude of the magnetic field becomes $10^{19} \mathrm{G}$, the soliton mass acquires approximately additional $150 \mathrm{MeV}$ by the external magnetic field.

Before we discuss the main results of the present work, we want to examine the values of the variational parameters for the profile functions. In Table I, we list their numerical results determined at the several selected values of the 


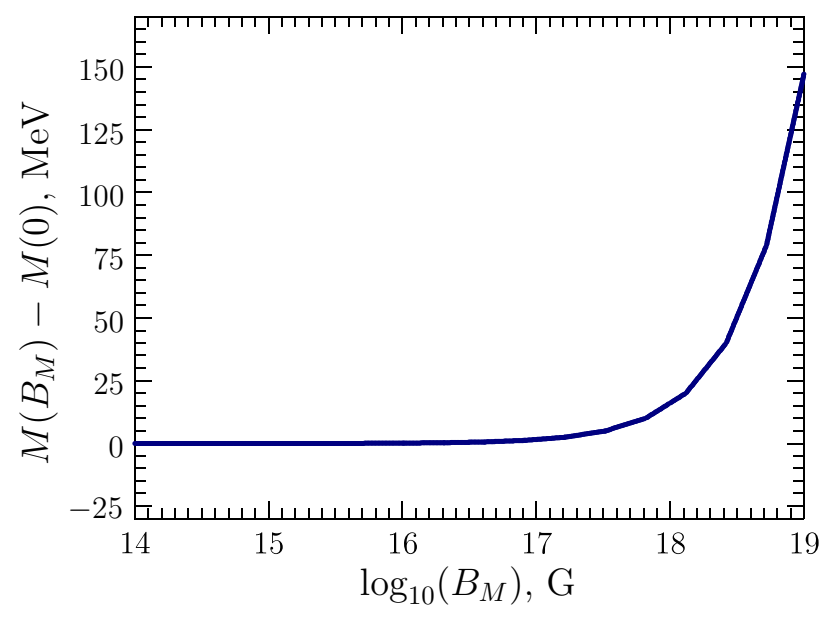

FIG. 1. The change of the classical soliton mass as a function of the external magnetic field given in the log scale. $M\left(B_{M}\right)$ and $M(0)$ denote respectively the values of the mass obtained with and without the magnetic field exerted.

magnetic field. Among the parameters presented in Table I, nonzero values of $\gamma_{n}$ 's are responsible for the deviation of the $P$ equisurfaces from the spherical form, whereas $\delta_{n}$ 's $(n>0)$ exhibit how the shape of the profile function is distorted from the spherically symmetric hedgehog form. One can see from Table I that at $B_{M}=10^{15} \mathrm{G}$, which characterizes the strength of the magnetic fields in magnetars, the $P$ equisurfaces already deviate from the spherically symmetric form.

It is interesting to observe that the value of $\beta_{1}$ is almost intact even at the upper limit of the strength of the magnetic fields in neutron stars $\left(\sim 10^{17} \mathrm{G}\right)$ [5]. However, if the strength of the magnetic field gets stronger, then its value is not zero anymore (see the corresponding value listed in the last column of Table I for $B_{M}=10^{19} \mathrm{G}$ ). In order to understand this behavior, we need to scrutinize the exponential term in Eq. (12). When $A$ in the first term is much larger than $\left(r q_{e} B_{M}\right)^{2}$ in the second one, for example, when $r^{2}\left(q_{e} \times 10^{17} \mathrm{G}\right)^{2} \sim 10 \mathrm{MeV}^{2} \ll\left(m_{\pi}^{2}+2 q_{e} B_{M} / 3\right) \approx$ $m_{\pi}^{2} \sim 0.18 \mathrm{GeV}^{2}$ numerically, then the asymptotic solution

TABLE I. Variational parameters for the profile functions $P$ and $\Theta$ at some selected values of the external magnetic field $B_{M}$.

\begin{tabular}{llrrl}
\hline \hline$B_{M}$ & \multicolumn{1}{c}{0} & $10^{15} \mathrm{G}$ & $10^{17} \mathrm{G}$ & \multicolumn{1}{c}{$10^{19} \mathrm{G}$} \\
\hline$r_{0}, \mathrm{fm}^{2}$ & 0.95646 & 0.95641 & 0.95200 & 0.97324 \\
$\beta_{0}$ & 1.31568 & 1.31554 & 1.30447 & 0.93320 \\
$\beta_{1}$ & 0 & 0 & 0 & 0.21958 \\
$\gamma_{2}, \mathrm{fm}^{2}$ & 0 & -0.64430 & 0.12305 & 0.33700 \\
$\gamma_{4}, \mathrm{fm}^{2}$ & 0 & 0.30370 & 0.21985 & 0.08227 \\
$\gamma_{6}, \mathrm{fm}^{2}$ & 0 & -0.10019 & -0.14775 & 0.21615 \\
$\delta_{0}, \mathrm{fm}^{-2}$ & 4.23604 & 3.90049 & 2.84256 & 3.21149 \\
$\delta_{1}, \mathrm{fm}$ & 0 & 0.13997 & 0.09016 & 0.9366 \\
$\delta_{2}, \mathrm{fm}$ & 0 & 0.24411 & 0.00207 & 0.00174 \\
\hline \hline
\end{tabular}

is not much influenced by the second term for the typical soliton size $(r \sim 1 \mathrm{fm})$. However, when the magnetic field is extremely strong, i.e., $B_{M} \sim 10^{19} \mathrm{G}$, we find $q_{e} B_{M}>m_{\pi}^{2}$. Thus, the second term dominates over the first one. It implies that the profile $P(r, \theta)$ will be shrunken by the second Gaussian term and $\beta_{1}$ is not zero anymore.

To understand the above-mentioned nature more clearly, we will delve into the baryon charge distribution of the axially deformed skyrmion, which is expressed as ${ }^{5}$

$$
B_{0}(r, \theta)=-\frac{P_{r} \Theta_{\theta}-P_{\theta} \Theta_{r}}{2 \pi^{2} r^{2}}\left(\frac{\sin \Theta}{\sin \theta}\right) \sin ^{2} P
$$

It will explicitly reveal how the soliton undergoes deformation in the presence of the strong magnetic field. In the Fig. 2(a), we depict the profiles of the baryon charge distributions along the $z$ direction $(\theta=0)$, while the Fig. 2(b) draws those in the perpendicular plane to the $z$ axis $(\theta=\pi / 2)$. Dotted curves correspond to the results with $B_{M}=0$, which should be spherically symmetric and are the same in both the left and right panels. We can take them as a reference for comparison. Taking the value of the magnetic field to be $B_{M}=10^{17} \mathrm{G}$, we see that the charge distribution of the soliton along the $z$ direction is deformed slightly, whereas it remains the same as that in the absence of magnetic field as shown in the right panel. If we take $B_{M}=10^{19} \mathrm{G}$, which can be realized in URHICs at the LHC, then the baryon charge distribution displays evidently the deformation of the soliton both along the $z$ direction and in the perpendicular plane to it.

To illuminate how the baryon charge distribution undergoes the change as the strength of the magnetic field is varied, we define the anisotropy of the baryon charge distribution as

$$
\Delta B_{0}(r) \equiv B_{0}(r, \pi / 2)-B_{0}(r, 0),
$$

where $B_{0}(r, \pi / 2)$ represents the baryon charge distribution in the perpendicular plane to the $z$ axis, and $B_{0}(r, 0)$ denotes that along the $z$ direction. Equation (17) shows how the isotropy of the baryon charge distribution is broken by the magnetic field. In the Fig. 3(a) we illustrate the results of $\Delta B_{0}$ as functions of $r$ and in the Fig. 3(b) those at $r=$ $0.2 \mathrm{fm}$ as a function of the magnetic field, respectively. The results of Fig. 3(a) clearly show that when $B_{M}=10^{17} \mathrm{G}$, which corresponds to the dashed curve, the soliton is more deformed along the $z$ direction than in the perpendicular plane to it. Moreover, it mainly occurs in the core part of the soliton. It implies that the baryon charge distribution will be taken slightly as a cigar-type form, since the results of $\Delta B_{0}(r)$ decreases in the core part. If one takes the stronger value of the magnetic field, i.e., $B_{M}=10^{19} \mathrm{G}$, then the

\footnotetext{
${ }^{5}$ See Eq. (8) above.
} 

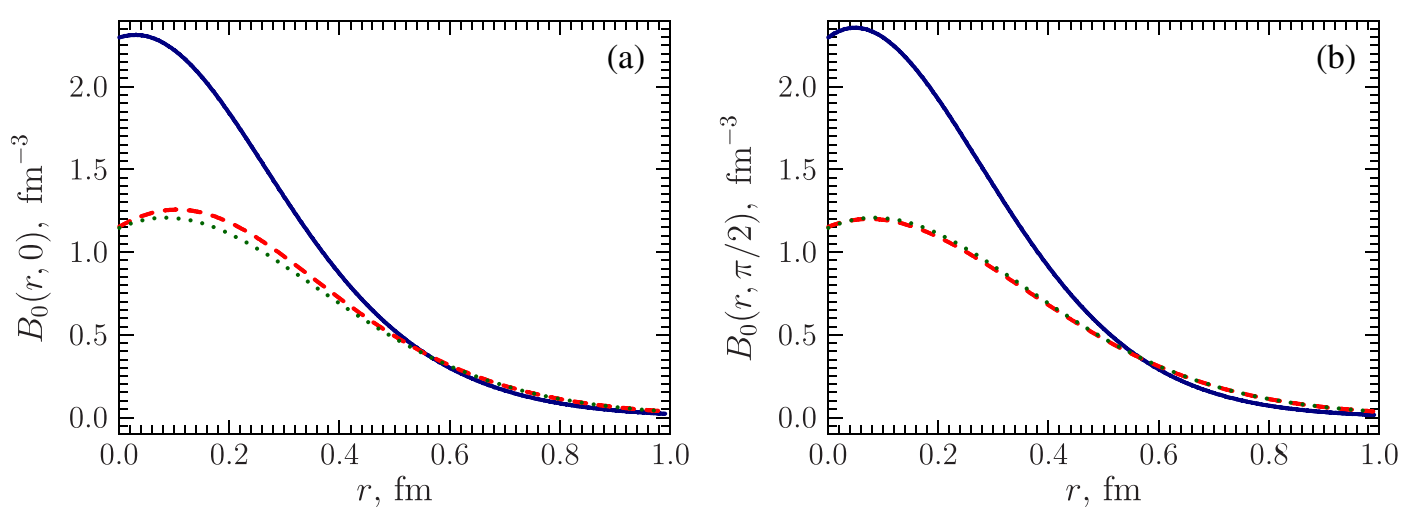

FIG. 2. The baryon charge distributions along the $z$ direction as functions of $r$ [in the left panel denoted by (a)] and those in the perpendicular plane to the $z$ axis as functions of $r$ [in the right panel denoted by (b)], respectively. The solid curves depict the results with $B_{M}=10^{19} \mathrm{G}$, the dashed ones draw those with $B_{M}=10^{17} \mathrm{G}$, and the dotted ones correspond to the case of $B_{M}=0$, respectively.
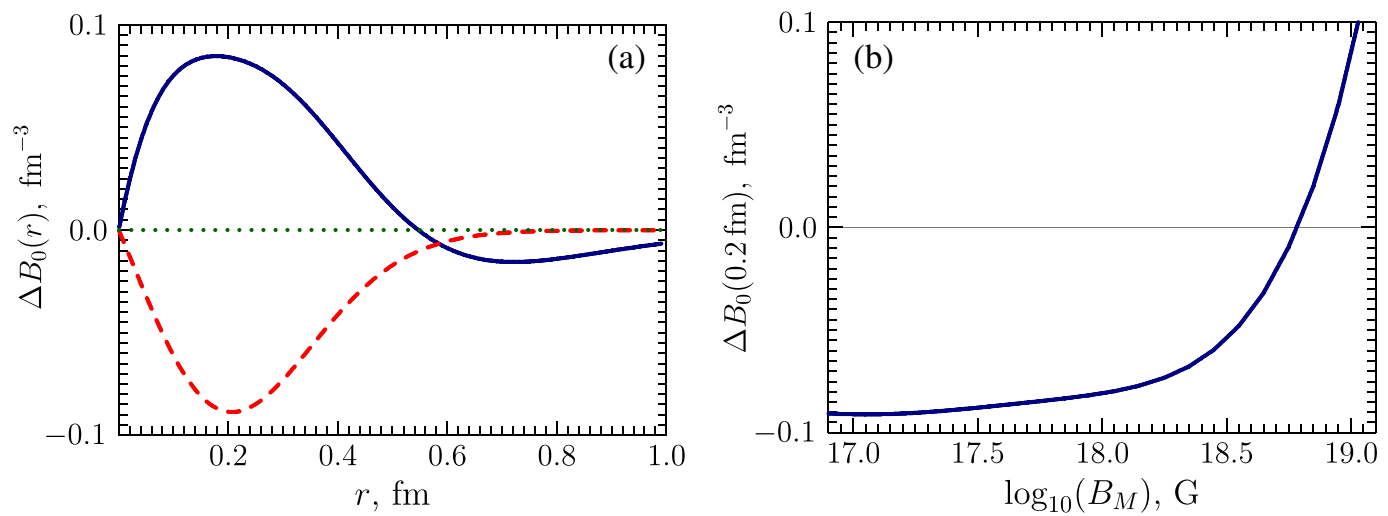

FIG. 3. In the left panel denoted by (a), the results of the anisotropy $\Delta B_{0}(r)$ defined in Eq. (17) as functions of $r$ and in the right panel denoted by (b) that of $\Delta B_{0}(0.2 \mathrm{fm})$ fixed at $r=0.2 \mathrm{fm}$ as a function of the magnetic field. Notations are the same as in Fig. 2.

baryon charge distribution is drastically changed from the previous case of $B_{M}=10^{17} \mathrm{G}$. The core part of the soliton undergoes the deformation in the $x y$ plane more strongly than along the $z$ direction. On the other hand, when it comes to its peripheral part, the situation is reversed. That is, while the peripheral shape of the soliton is less distorted than in the core part, the soliton is slightly more deformed along the $z$ direction in comparison with that in the perpendicular plane to it.

To see the process of the soliton deformation more closely, we scrutinize $\Delta B_{0}$ at a fixed value of $r$, for example, at $r=0.2 \mathrm{fm}$, as the $B_{M}$ field varied from $10^{17} \mathrm{G}$ to $10^{19} \mathrm{G}$. The corresponding result is illustrated in the Fig. 3(b). When the strength of the magnetic field is given between $10^{17} \mathrm{G}$ and $10^{18} \mathrm{G}$, we can clearly observe that the core part of the soliton is more deformed in the $x y$ plane, compared with that along the $z$ direction. However, if we further increase the strength of the magnetic field close to $10^{19} \mathrm{G}$, the situation becomes other way around, i.e., the core part of the soliton is deformed more strongly along the $z$ direction in comparison with that in the perpendicular plane to it.
In general, the baryon charge distribution is more compactly deformed in the presence of the strong magnetic field. This can be observed by comparing the solid curves with dotted ones in the Fig. 2. Since the quadratic term with regards to $B_{M}$ in Eq. (12) come into dominant play when the magnetic field is very strong. In fact, this is related to the quadratic term like a harmonic oscillator potential in the approximated differential equation given in Eq. (9) in the asymptotic limit, which plays effectively a role of a confining potential that arises from the strong magnetic field. The physical implications of this confining potential are that the pions are localized and are forced to be confined by the external strong magnetic field.

Here, it is necessary to remind that the baryon is a topological object made of the nonlinearly interacting pions. In this context, although the Skyrme model has no explicit quark degrees of freedom, the obvious charged pion localizations due to the external magnetic field will localize also the neutral pions by means of the nonlinear interactions. Moreover, the quantization by rotation in isospin space infers that both the charged and neutral pions are under the influence of the strong magnetic field. It can 
be explicitly seen from the expressions of the baryon charge distribution (16) and the mass functional [see Eqs. (A2) and (A3)], which do not distinguish the charged components of the pion fields. ${ }^{6}$

We can examine the corresponding localizations of the nonlinearly interacting pions by considering the baryon charge distribution within a certain region. For example, we integrate the baryon charge distribution up to $1 \mathrm{fm}$

$$
B_{(1 \mathrm{fm})}=\int_{0}^{1 \mathrm{fm}} r^{2} d r \int d \Omega B_{0}
$$

with the magnetic field varied. Then, comparing the results with different values of $B_{M}$, we can see how the charged pions are forced toward the core region inside a nucleon. Taking three different values of $B_{M}$, we obtain the following results: $B_{(1 \mathrm{fm})}=0.9014$ for $B_{M}=0, B_{(1 \mathrm{fm})}=0.9024$ for $B_{M}=10^{17} \mathrm{G}$ and $B_{(1 \mathrm{fm})}=0.9665$ for $B_{M}=10^{19} \mathrm{G}$, respectively. ${ }^{7}$ The comparison of these values indicates that the baryon charge distribution is indeed squeezed into the core region due to the localization of the charged pions.

\section{QUANTIZATION OF THE SPHEROIDAL SOLITON}

We are now in a position to discuss the quantization of the axially deformed soliton, i.e., the spheroidal one and the relevant results. The quantization of a spherically symmetric chiral soliton is generally performed by introducing the zero-mode quantization with the collective coordinates introduced [37]. As we already discussed in the previous section, the spherical symmetry of the soliton is already broken in the presence of the magnetic field. However, we still have an axial symmetry as presented in Eqs. (5)-(7). Thus, we consider independent rotations in the coordinate and isospin spaces as follows

$$
P=P\left(\mathcal{R}^{-1}(t) \boldsymbol{r}\right), \quad \boldsymbol{N}=\mathcal{I}(t) \boldsymbol{N}\left(\mathcal{R}^{-1}(t) \boldsymbol{r}\right),
$$

where $\mathcal{R}$ and $\mathcal{I}$ represent the SO (3) rotational and isorotational matrices, respectively. Having carried out these slow time-dependent rotations and performed the spatial integration, we arrive at a collective Lagrangian

$$
\begin{aligned}
L= & -M+\frac{\omega_{1}^{2}+\omega_{2}^{2}}{2} \Lambda_{\omega \omega, 12}-\left(\omega_{1} \Omega_{1}+\omega_{2} \Omega_{2}\right) \Lambda_{\omega \Omega, 12} \\
& +\frac{\Omega_{1}^{2}+\Omega_{2}^{2}}{2} \Lambda_{\Omega \Omega, 12}+\frac{\left(\omega_{3}-\Omega_{3}\right)^{2}}{2} \Lambda_{\omega \Omega, 33} .
\end{aligned}
$$

\footnotetext{
${ }^{6}$ There is yet another effect of the magnetic field on the quarks inside the neutral pion trough the wave function deformations. This effect comes from higher-order corrections with respect to the external magnetic field, which is not considered in this work.

Of course, we get $B=1$ in all cases if one integrates properly all over the region, as it should be.
}

Here $\omega_{i}$ and $\Omega_{i}$ denote the angular velocities in isospin and coordinate spaces, respectively. The explicit expressions of the functionals $\Lambda[P, \Theta]$ can be found in the Appendix.

Defining the canonical conjugate variables in the bodyfixed reference system as

$$
T_{i}=\frac{\partial L}{\partial \omega_{i}} \quad \text { and } \quad J_{i}=\frac{\partial L}{\partial \Omega_{i}}
$$

we derive from the time-dependent Lagrangian in Eq. (20) the collective Hamiltonian as

$$
\begin{aligned}
\hat{H}= & M+\frac{\hat{T}_{3}^{2}}{2 \Lambda_{\omega \Omega, 33}}+\frac{\left(\hat{T}_{1} \hat{J}_{1}+\hat{T}_{2} \hat{J}_{2}\right) \Lambda_{\omega \Omega, 12}}{\Lambda_{\omega \omega, 12} \Lambda_{\Omega \Omega, 12}-\Lambda_{\omega \Omega, 12}^{2}} \\
& +\frac{\left(\hat{T}_{1}^{2}+\hat{T}_{2}^{2}\right) \Lambda_{\Omega \Omega, 12}+\left(\hat{J}_{1}^{2}+\hat{J}_{2}^{2}\right) \Lambda_{\omega \omega, 12}}{2\left(\Lambda_{\omega \omega, 12} \Lambda_{\Omega \Omega, 12}-\Lambda_{\omega \Omega, 12}^{2}\right)} .
\end{aligned}
$$

Diagonalizing the Hamiltonian of Eq. (22), we obtain the baryon eigenstates $\left|T, T_{3} ; J, J_{3}\right\rangle$ and the energies of the axially deformed nucleon and the $\Delta$ isobar:

$$
\begin{aligned}
E= & M+\frac{T_{3}^{2}}{2 \Lambda_{\omega \Omega, 33}} \\
& +\frac{\Lambda_{\Omega \Omega, 12}+\Lambda_{\omega \omega, 12}-2 \Lambda_{\omega \Omega, 12}}{2\left(\Lambda_{\omega \omega, 12} \Lambda_{\Omega \Omega, 12}-\Lambda_{\omega \Omega, 12}^{2}\right)}\left(T(T+1)-T_{3}^{2}\right) .
\end{aligned}
$$

From the third term of Eq. (23), one observes that in the presence of the external magnetic field the degeneracy in the energy between the different isospin states of the $\Delta$ isobar are partially lifted. For example, the proton and neutrons are still in degeneracy, i.e., $m_{\mathrm{p}}=m_{\mathrm{n}}$, while the $\Delta$ isobar isospin states are partially split, i.e., $m_{\Delta^{++}}=m_{\Delta^{-}} \neq$ $m_{\Delta^{+}}=m_{\Delta^{0}}$.

The results for the masses of baryons at certain values of the magnetic field $B_{M}$ are listed in Table II. As in the case of the classical soliton, the masses of the nucleons and $\Delta$ isobars are almost intact till the strength of the magnetic field is reached at around $10^{17} \mathrm{G}$. Keeping in mind that the magnetic field in magnetars is approximately $B_{M}=10^{15} \mathrm{G}$, the baryon masses are almost not changed. However, if one further increases the strength of $B_{M}$, the masses of all the nucleons and $\Delta$ isobars start to grow. At $B_{M}=10^{17} \mathrm{G}$ the change of the baryon mass is already not negligible. Then, when it is reached to $B_{M}=10^{19} \mathrm{G}$, the masses increase by

TABLE II. Masses of baryons at some selected values of the external magnetic field $B_{M}$. All masses are given in units of $\mathrm{MeV}$.

\begin{tabular}{lrrrr}
\hline \hline$B_{M}$ & \multicolumn{1}{c}{0} & \multicolumn{1}{c}{$10^{15} \mathrm{G}$} & \multicolumn{1}{c}{$10^{17} \mathrm{G}$} & \multicolumn{1}{c}{$10^{19} \mathrm{G}$} \\
\hline$m_{\mathrm{n}, \mathrm{p}}$ & 939.8035 & 939.8212 & 941.5769 & 1113.4133 \\
$m_{\Delta^{++}, \Delta^{-}}$ & 1233.6770 & 1233.6951 & 1236.5624 & 1530.4224 \\
$m_{\Delta^{+}, \Delta^{0}}$ & 1233.6770 & 1233.6949 & 1236.3618 & 1507.7573 \\
\hline \hline
\end{tabular}




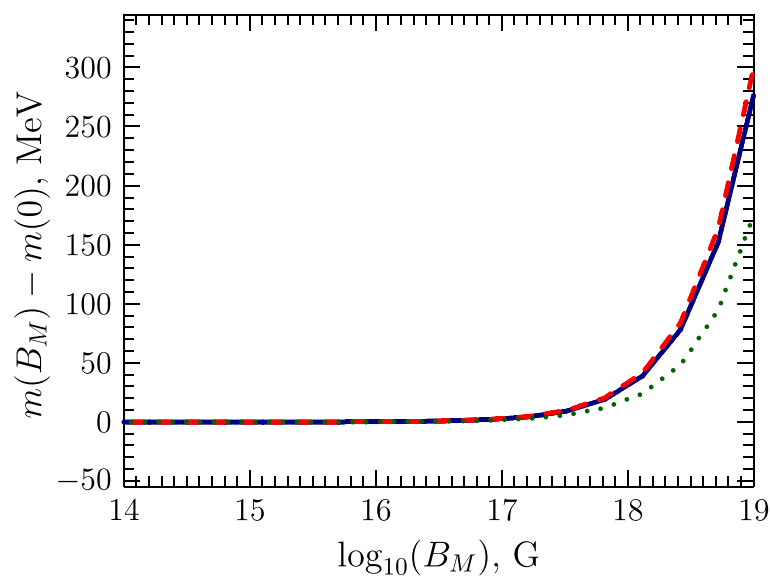

FIG. 4. The changes of the baryon masses as a function of the magnetic field. The solid curve depicts $m_{\Delta^{0}}$, whereas the dashed one draws $m_{\Delta^{-}}$. The dotted one represents $m_{\mathrm{n}}$, respectively.

about $15-20 \%$. Note that $\Delta$-isobar states actually remain degenerate even though the magnetic field gets very strong. Only at very large values of the magnetic field, the degeneracy of the $\Delta$ isobars will be partially lifted as discussed above.

In Fig. 4 we show how the masses of the baryons will be changed as $B_{M}$ increases. The results look very similar to the change of the classical soliton mass as shown in Fig. 1. However, the rates of the increment in the masses of the baryons are still different. The reason can be found in the changes of the moments of inertia. ${ }^{8}$ Since the soliton is deformed in the presence of the strong magnetic fields, the magnitudes of the moments of inertia are decreased. It indicates that not only the baryon charge distribution is changed but also the mass distribution inside the soliton becomes more compact in the presence of the strong magnetic field than in free space. As was done in the case of the baryon charge distribution, we can consider the integrate value of the mass distribution up to $1 \mathrm{fm}$ [see Eq. (18)]. Then we obtain the results at three different values of $B_{M}$ as follows: $M_{(1 \mathrm{fm})}=0.818 M$ for $B_{M}=0$, $M_{(1 \mathrm{fm})}=0.820 M$ for $B_{M}=10^{17} \mathrm{G}$ and $M_{(1 \mathrm{fm})}=0.911 M$ for $B_{M}=10^{19} \mathrm{G}$. This indicates that the masses of the baryons tend to be more compact in the presence of the magnetic fields than in free space.

It is also very interesting to examine the moments of inertia for the spheroidal solitons. We first define the following quantities

$$
\begin{aligned}
\Delta m_{(0,-)}\left(B_{M}\right)= & {\left[m_{\Delta^{0}}\left(B_{M}\right)-m_{\Delta^{-}}\left(B_{M}\right)\right] } \\
& -\left[m_{\Delta^{0}}(0)-m_{\Delta^{-}}(0)\right],
\end{aligned}
$$

\footnotetext{
${ }^{8}$ The formula for the moments of inertia, see Eq. (A5).
}

$$
\begin{aligned}
\Delta m_{(0, \mathrm{n})}\left(B_{M}\right)= & {\left[m_{\Delta^{0}}\left(B_{M}\right)-m_{\mathrm{n}}\left(B_{M}\right)\right] } \\
& -\left[m_{\Delta^{0}}(0)-m_{\mathrm{n}}(0)\right], \\
\Delta m_{(-, \mathrm{n})}\left(B_{M}\right)= & {\left[m_{\Delta^{-}}\left(B_{M}\right)-m_{\mathrm{n}}\left(B_{M}\right)\right] } \\
& -\left[m_{\Delta^{-}}(0)-m_{\mathrm{n}}(0)\right] .
\end{aligned}
$$

They describe how much the mass splittings of the baryons undergo the changes in the presence of the magnetic field. The results are illustrated in Fig. 5. Here we explicitly demonstrate that the moments of inertia decrease, which bring about the rise of the $\Delta-N$ mass splittings, which are illustrated in the dashed and dotted curves in Fig. 5. One can also observe that the mass degeneracy in the different isospin states of the $\Delta$ isobars is lifted, as shown in the solid curve of Fig. 5. While the degeneracy is more or less kept to be intact till $B_{M}=10^{17} \mathrm{G}$, it starts to be removed. If $B_{M}$ continues to increase, the splitting between the $\Delta^{0}$ and $\Delta^{-}$ masses becomes prominent.

Finally, we want to mention that there is still a caveat that is related to the strong magnetic fields. A novel feature emerges when the magnetic field is very strong, called the Paschen-Back (PB) effect [53]. Originally, the PB effect arises when the strength of the magnetic field dominates over the spin-orbit coupling of an atomic system. In the presence of the weak magnetic field, all the eigenstates of an atom are split, which is known as the anomalous Zeeman effect. However, if the magnetic field is so strong that it overcomes the spin-orbit interaction, then the spherical symmetry is completely broken, so that the total angular momentum squared, $J^{2}$, is no more a good quantum number but $L_{z}$ and $S_{z}$ are the good quantum numbers [54]. However, we still have cylindrical symmetry or axial symmetry in the presence of the constant external magnetic field along a specific direction as discussed in this work.

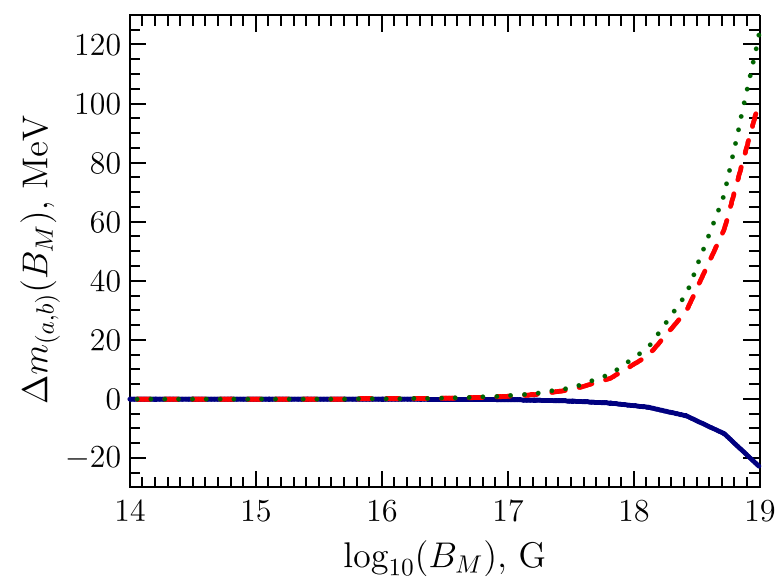

FIG. 5. The change of the baryon mass splittings in the presence of the magnetic field. The solid curve draws the result of $\Delta m_{(0,-)}\left(B_{M}\right)$, whereas the dashed one depicts $\Delta m_{(0, \mathrm{n})\left(\mathrm{B}_{\mathrm{M}}\right)}$. The dotted one shows $\Delta m_{(-, \mathrm{n})}\left(\mathrm{B}_{\mathrm{M}}\right)$. For the definitions of $\Delta m_{(a, b)}$, see Eqs. (25)-(26). 
Thus, $2(2 l+1)$ degeneracy in $m_{l}+m_{s}$ will appear. This is called the PB effect. In fact, Iwasaki et al. discussed the PB effect [55], when the strong magnetic field $\left(\sim 10^{19} \mathrm{G}\right)$ is exerted on a charmonium system. They found a very interesting feature: The strong magnetic field induces mixing between $S=0$ and $S=1$ states. This may lead to the mixing between the $\eta_{c}$ and $J / \psi$ in $S=1$ and $S_{z}=0$ states. It implies that when the magnetic field is very strong, one can expect the same phenomena in a baryonic system such as the mixing between the proton with $S=1 / 2$ and $S_{z}=1 / 2$ and the $\Delta^{+}$isobar with $S=3 / 2$ and $S_{z}=1 / 2$. We will investigate this important physics elsewhere.

\section{SUMMARY AND OUTLOOK}

In the present work, we investigated how the nucleons and $\Delta$ isobars undergo the deformation in the presence of the strong magnetic field within the framework of the Skyrme model. We first examined the changes of the classical soliton under the influence of the strong magnetic field. The mass of the classical soliton remains unchanged till the magnitude of the magnetic field reached $10^{17} \mathrm{G}$. However, if the magnetic field gets stronger than this value, the mass starts to increase. The soliton is deformed in a rather nontrivial way as the strength of the magnetic field varied. We exhibited explicitly and thoroughly how the soliton properties were changed as the magnetic field was altered. When the magnitude of the magnetic field is $10^{17} \mathrm{G}$, the soliton was deformed more strongly along the $z$ direction than in the perpendicular plane to it. The core part of the soliton was mainly modified, which indicates that the shape of the soliton will turn to a cigar-type form. If the value magnetic field was taken to be $10^{19} \mathrm{G}$, then the baryon charge distribution was drastically altered. The core part of the soliton was deformed more strongly in the $x y$ plane than along the $z$ direction. On the contrary, the peripheral shape of the soliton was less distorted than in the core part, whereas the soliton was slightly more deformed along the $z$ direction than in the perpendicular plane to it.

We performed the zero-mode quantization of the spheroidal soliton in the presence of the magnetic field. We found that the solitonic moments of inertia decreases as the magnetic field increases. It means that the masses of the nucleon and $\Delta$ isobar should get larger. Moreover, we observed that $\Delta$-N mass splitting also increases. The spherical nucleon in free space was deformed into a cigar-type form when the magnetic field was present. The case of the $\Delta$ isobars was similar to the nucleon case but their masses increased slightly more than the nucleon did as the magnetic field is strengthened. We found that the mass of $\Delta^{++}$is degenerate with that of $\Delta^{-}$, whereas $\Delta^{+}$has the same mass as $\Delta^{0}$. However, the mass degeneracy was partially lifted.

From the present work, we conclude that there is no need to consider the effects of the strong magnetic field in analyzing the equation of the states (EoS) at high densities that may exist in interiors of compact stellar objects, since the nucleon masses are almost intact till the magnitude of the magnetic field reaches $10^{17} \mathrm{G}$. It is interesting to see that one can make the similar conclusion from the recent studies on the EoS of strongly magnetized quark matter within the Nambu-Jona-Lasinio model [56]. However, when it comes to ultrarelativistic heavy ion collisions at the LHC, it is of great significance to take into accounts the effects coming from the strong magnetic field. This will lead to nontrivial consequences. Furthermore, generalizations of the model may be performed by including the explicit isospin breaking effects in the mesonic sector in order to study the changes in the neutron-proton mass difference under the influence of the external magnetic field. The Paschen-Beck effects on baryonic systems are yet another interesting issue, which can be investigated as future works. The relevant works are under way.

\section{ACKNOWLEDGMENTS}

We are grateful to P. Gubler, A. Hosaka, K. Itakura, T. Maruyama for useful discussions. U. Y. and H.-Ch. K. would like to express their gratitude to the members of the Advanced Science Research Center at Japan Atomic Energy Agency for the hospitality, where the present work was done. This work is supported by the Basic Science Research Program through the National Research Foundation (NRF) of Korea funded by the Korean government (Ministry of Education, Science and Technology, MEST), No. 2016R1D1A1B03935053 (UY) and No. 2018R1A2B2001752 (HChK).

\section{APPENDIX: MASS AND MOMENTS OF INERTIA OF THE SPHEROIDAL SOLITON}

For convenience, we introduce the following shorthanded notations:

$$
\begin{aligned}
P_{r} & \equiv \partial_{r} P, \quad P_{\theta} \equiv \partial_{\theta} P, \quad \Theta_{r} \equiv \partial_{r} \Theta, \quad \Theta_{\theta} \equiv \partial_{\theta} \Theta \\
S_{P} & \equiv \sin P, \quad C_{P} \equiv \cos P, \quad S_{\Theta} \equiv \sin \Theta, \\
C_{\Theta} & \equiv \cos \Theta, \quad s_{\theta} \equiv \sin \theta, \quad c_{\theta} \equiv \cos \theta .
\end{aligned}
$$

The classical soliton mass $M$ in the Lagrangian in Eq. (20) and the explicit change of the soliton mass $\Delta M$ in the external magnetic field are expressed as follows:

$$
\begin{aligned}
M= & \pi \int_{0}^{\infty} d r r^{2} \int_{0}^{\pi} s_{\theta} d \theta\left\{\frac { F _ { \pi } ^ { 2 } } { 4 r ^ { 2 } } \left[P_{\theta}^{2}+r^{2} P_{r}^{2}\right.\right. \\
& \left.+S_{P}^{2}\left(\frac{S_{\Theta}^{2}}{s_{\theta}^{2}}+\Theta_{\theta}^{2}+r^{2} \Theta_{r}^{2}\right)\right]+\frac{S_{P}^{2}}{e^{2} r^{4}}\left[\frac{S_{\Theta}^{2}}{s_{\theta}^{2}}\left(P_{\theta}^{2}+r^{2} P_{r}^{2}\right)\right. \\
& \left.+S_{P}^{2} \frac{S_{\Theta}^{2}}{s_{\theta}^{2}}\left(\Theta_{\theta}^{2}+r^{2} \Theta_{r}^{2}\right)+r^{2}\left(P_{r} \Theta_{\theta}-P_{\theta} \Theta_{r}\right)^{2}\right] \\
& \left.+\frac{m_{\pi}^{2} F_{\pi}^{2}}{2}\left(1-C_{P}\right)\right\}+\Delta M,
\end{aligned}
$$




$$
\begin{aligned}
\Delta M= & \pi \int_{0}^{\infty} d r r^{2} \int_{0}^{\pi} s_{\theta} d \theta\left\{\frac{F_{\pi}^{2}}{16}\right. \\
& +\frac{1}{4 e^{2} r^{2}}\left(P_{\theta}^{2}+r^{2} P_{r}^{2}+S_{P}^{2}\left(\Theta_{\theta}^{2}+r^{2} \Theta_{r}^{2}\right)\right\} \\
& \times q_{e} B_{M}\left(4+q_{e} B_{M} r^{2} s_{\theta}^{2}\right) S_{P}^{2} S_{\Theta}^{2} .
\end{aligned}
$$

The generic form for the moment of inertia is defined as

$$
\Lambda=2 \pi \int_{0}^{\infty} d r r^{2} \int_{0}^{\pi} s_{\theta} d \theta \lambda
$$

where the contributions from the different parts of the Lagrangian (20) are given as

$$
\begin{aligned}
\lambda_{\omega \omega, 12}= & \Delta \lambda_{\omega \omega, 12}+\frac{F_{\pi}^{2}}{8}\left(1+C_{\Theta}^{2}\right) S_{P}^{2} \\
& +\frac{S_{P}^{2}}{2 e^{2} r^{2}}\left[\left(1+C_{\Theta}^{2}\right)\left(P_{\theta}^{2}+r^{2} P_{r}^{2}\right)\right. \\
& \left.+S_{P}^{2}\left(\frac{S_{\Theta}^{2}}{s_{\theta}^{2}}+C_{\Theta}^{2}\left(\Theta_{\theta}^{2}+r^{2} \Theta_{r}^{2}\right)\right)\right], \\
\lambda_{\omega \Omega, 12}= & \Delta \lambda_{\omega \Omega, 12}+\frac{F_{\pi}^{2}}{8}\left(c_{\theta} C_{\Theta} \frac{S_{\Theta}}{s_{\theta}}+\Theta_{\theta}\right) S_{P}^{2} \\
& +\frac{S_{P}^{2}}{2 e^{2} r^{2}}\left[c_{\theta} C_{\Theta} \frac{S_{\Theta}}{c_{\theta}}\left(P_{\theta}^{2}+r^{2} P_{r}^{2}+S_{P}^{2}\left(\Theta_{\theta}^{2}+r^{2} P_{r}^{2}\right)\right)\right. \\
& \left.+S_{P}^{2} S_{\Theta}^{2} s_{\theta}^{-2} \Theta_{\theta}+r^{2} P_{r}\left(P_{r} \Theta_{\theta}-\Theta_{r} P_{\theta}\right)\right],
\end{aligned}
$$

$$
\begin{aligned}
\lambda_{\Omega \Omega, 12}= & \Delta \lambda_{\Omega \Omega, 12}+\frac{F_{\pi}^{2}}{8}\left[P_{\theta}^{2}+S_{P}^{2}\left(c_{\theta}^{2} \frac{S_{\Theta}^{2}}{s_{\theta}^{2}}+\Theta_{\theta}^{2}\right)\right] \\
& +\frac{S_{P}^{2}}{2 e^{2} r^{2}}\left[\frac { S _ { \Theta } ^ { 2 } } { s _ { \theta } ^ { 2 } } \left(\left(1+c_{\theta}^{2}\right)\left(P_{\theta}^{2}+S_{P}^{2} \Theta_{\theta}^{2}\right)\right.\right. \\
& \left.\left.+r^{2}\left(P_{r}^{2}+S_{P}^{2} \Theta_{r}^{2}\right) c_{\theta}^{2}\right)+r^{2}\left(P_{r} \Theta_{\theta}-P_{\theta} \Theta_{r}\right)^{2}\right],
\end{aligned}
$$

and one can also note, that the moment of inertia corresponding to the quantization axis does not depend explicitly on the magnetic field

$\lambda_{\omega \Omega, 33}=\frac{F_{\pi}^{2}}{4} S_{\Theta}^{2} S_{P}^{2}+\frac{S_{P}^{2}}{e^{2} r^{2}} S_{\Theta}^{2}\left(P_{\theta}^{2}+r^{2} P_{r}^{2}+S_{P}^{2}\left(\Theta_{\theta}^{2}+r^{2} \Theta_{r}^{2}\right)\right)$.

Finally, the additional parts of the moments of inertia arising from the external magnetic field are expressed as

$\Delta \lambda_{\omega \omega, 12}=\frac{q_{e} B_{M}}{4 e^{2}}\left(4+q_{e} B_{M} r^{2} s_{\theta}^{2}\right) S_{P}^{4} S_{\Theta}^{2}$,

$\Delta \lambda_{\omega \Omega, 12}=\frac{q_{e} B_{M}}{4 e^{2}}\left(4+q_{e} B_{M} r^{2} s_{\theta}^{2}\right) S_{P}^{4} S_{\Theta}^{2} \Theta_{\theta}$,

$\Delta \lambda_{\Omega \Omega, 12}=\frac{q_{e} B_{M}}{4 e^{2}}\left(4+q_{e} B_{M} r^{2} s_{\theta}^{2}\right) S_{P}^{2} S_{\Theta}^{2}\left(P_{\theta}^{2}+S_{P}^{2} \Theta_{\theta}^{2}\right)$.
[1] S. Mereghetti, J. Pons, and A. Melatos, Space Sci. Rev. 191, 315 (2015).

[2] V. M. Kaspi and A. Beloborodov, Annu. Rev. Astron. Astrophys. 55, 261 (2017).

[3] C. Kouveliotou et al., Nature (London) 393, 235 (1998).

[4] P. Bhattacharjee and G. Sigl, Phys. Rep. 327, 109 (2000).

[5] C. Thompson and R. C. Duncan, Astrophys. J. 473, 322 (1996).

[6] J. Rafelski and B. Muller, Phys. Rev. Lett. 36, 517 (1976).

[7] V. Skokov, A. Y. Illarionov, and V. Toneev, Int. J. Mod. Phys. A 24, 5925 (2009).

[8] X. G. Huang, Rep. Prog. Phys. 79, 076302 (2016).

[9] D. E. Kharzeev, L. D. McLerran, and H. J. Warringa, Nucl. Phys. A803, 227 (2008).

[10] V. Voronyuk, V. D. Toneev, W. Cassing, E. L. Bratkovskaya, V. P. Konchakovski, and S. A. Voloshin, Phys. Rev. C 83, 054911 (2011).

[11] L. Ou and B. A. Li, Phys. Rev. C 84, 064605 (2011).
[12] J. Bloczynski, X. G. Huang, X. Zhang, and J. Liao, Nucl. Phys. A939, 85 (2015).

[13] W. T. Deng and X. G. Huang, Phys. Lett. B 742, 296 (2015).

[14] K. Hattori and X. G. Huang, Nucl. Sci. Tech. 28, 26 (2017).

[15] X. L. Zhao, Y. G. Ma, and G. L. Ma, Phys. Rev. C 97, 024910 (2018).

[16] Y. Hidaka and A. Yamamoto, Phys. Rev. D 87, 094502 (2013).

[17] C. S. Machado, F. S. Navarra, E. G. de Oliveira, J. Noronha, and M. Strickland, Phys. Rev. D 88, 034009 (2013).

[18] J. Alford and M. Strickland, Phys. Rev. D 88, 105017 (2013).

[19] C. S. Machado, S. I. Finazzo, R. D. Matheus, and J. Noronha, Phys. Rev. D 89, 074027 (2014).

[20] E. V. Luschevskaya, O. E. Solovjeva, O. A. Kochetkov, and O. V. Teryaev, Nucl. Phys. B898, 627 (2015).

[21] H. Taya, Phys. Rev. D 92, 014038 (2015). 
[22] C. Bonati, M. D’Elia, and A. Rucci, Phys. Rev. D 92, 054014 (2015).

[23] P. Gubler, K. Hattori, S. H. Lee, M. Oka, S. Ozaki, and K. Suzuki, Phys. Rev. D 93, 054026 (2016).

[24] K. Suzuki and T. Yoshida, Phys. Rev. D 93, 051502 (2016).

[25] E. V. Luschevskaya, O. A. Kochetkov, O. V. Teryaev, and O. E. Solovjeva, JETP Lett. 101, 674 (2015).

[26] M. A. Andreichikov, B. O. Kerbikov, E. V. Luschevskaya, Y. A. Simonov, and O. E. Solovjeva, J. High Energy Phys. 05 (2017) 007.

[27] H. Liu, X. Wang, L. Yu, and M. Huang, Phys. Rev. D 97, 076008 (2018).

[28] M. Coppola, D. Gómez Dumm, and N. N. Scoccola, Phys. Lett. B 782, 155 (2018).

[29] S. S. Avancini, R. L. S. Farias, and W. R. Tavares, Phys. Rev. D 99, 056009 (2019).

[30] S. S. Avancini, R. L. S. Farias, M. Benghi Pinto, W. R. Tavares, and V. S. Timóteo, Phys. Lett. B 767, 247 (2017).

[31] M. A. Andreichikov, B. O. Kerbikov, V. D. Orlovsky, and Y. A. Simonov, Phys. Rev. D 89, 074033 (2014).

[32] M. Bigdeli, Phys. Rev. C 95, 024309 (2017).

[33] E. Witten, Nucl. Phys. B160, 57 (1979).

[34] E. Witten, Nucl. Phys. B223, 422 (1983).

[35] E. Witten, Nucl. Phys. B223, 433 (1983).

[36] T. H. R. Skyrme, Proc. R. Soc. A 260, 127 (1961).

[37] G. S. Adkins, C. R. Nappi, and E. Witten, Nucl. Phys. B228, 552 (1983).

[38] H.-Ch. Kim, P. Schweitzer, and U. T. Yakhshiev, Phys. Lett. B 718, 625 (2012).

[39] U. T. Yakhshiev and H.-Ch. Kim, Phys. Lett. B 726, 375 (2013).
[40] J. H. Jung, U. T. Yakhshiev, and H.-Ch. Kim, Phys. Lett. B 723, 442 (2013).

[41] J. H. Jung, U. Yakhshiev, H.-Ch. Kim, and P. Schweitzer, Phys. Rev. D 89, 114021 (2014).

[42] J. H. Jung, U. Yakhshiev, and H.-Ch. Kim, Phys. Rev. D 93, 054016 (2016).

[43] K. H. Hong, U. Yakhshiev, and H.-Ch. Kim, arXiv: 1806.06504.

[44] U. T. Yakhshiev, M. M. Musakhanov, A. M. Rakhimov, U. G. Meissner, and A. Wirzba, Nucl. Phys. A700, 403 (2002).

[45] U. T. Yakhshiev, Phys. Rev. C 88, 034318 (2013).

[46] U. G. Meissner, A. M. Rakhimov, A. Wirzba, and U.T. Yakhshiev, Eur. Phys. J. A 36, 37 (2008).

[47] U. G. Meissner, A. M. Rakhimov, A. Wirzba, and U.T. Yakhshiev, EPJ Web Conf. 3, 06008 (2010).

[48] G. Steigman, Int. J. Mod. Phys. E 15, 1 (2006).

[49] R. H. Cyburt, Phys. Rev. D 70, 023505 (2004).

[50] G. S. Adkins and C. R. Nappi, Nucl. Phys. B233, 109 (1984).

[51] J. Gasser and H. Leutwyler, Nucl. Phys. B250, 465 (1985).

[52] T. E. Rudy, H. W. Fearing, and S. Scherer, Phys. Rev. C 50, 447 (1994).

[53] F. Paschen and E. Back, Physica 1, 261 (1921).

[54] J. J. Sakurai and J. Napolitano, Modern Quantum Mechanics, 2nd ed. (Cambridge University Press, Cambridge, England, 2017).

[55] S. Iwasaki, M. Oka, K. Suzuki, and T. Yoshida, Phys. Lett. B 790, 71 (2019).

[56] S. S. Avancini, V. Dexheimer, R. L. S. Farias, and V. S. Timóteo, Phys. Rev. C 97, 035207 (2018). 